widely-travelled birdwatcher. Perhaps, but I will find it more useful as a quick reference for identifying unfamiliar bird names encountered in the serial literature, and in this respect its 59-page index is admirable.

Specialists in particular bird groups will not find it hard to quibble with points of detail, but on the whole the compilers offer a reasonable compromise between the often conflicting treatments to be found in avifaunal publications. Several other books of this type have appeared since 1974 , the present one being the most comprehensive and therefore the most costly. It is the only one to include subspecies; but this is the weakest aspect of the book, since its authors are not taxonomists. A simplified listing of racial names gives a misleading impression of subspecific rigidity (whereas clinal situations are common even within land areas as small as the British Isles); moreover, named races present a continuum from welldifferentiated semi-species to those hardly worthy of recognition, though this basic truth is not made evident to the reader. Thus, this is a potentially useful book, but not a definitive work.

Robert Hudson is on the scientific staff of the British Trust for Ornithology, and is editor of the Trust's quarterly journal Bird Study.

\title{
Semiconductor lasers come of age
}

\section{S. D. Smith}

Physics of Semiconductor Laser Devices. By G. H. B. Thompson. Pp.549. (Wiley: 1980.) £22.75, \$68.25.

THE semiconductor diode laser based upon the radiative recombination of carriers injected across a $\mathrm{p}-\mathrm{n}$ junction is now nearly 20 years old from the date of the original concept. It has developed from a laboratory speciality operating only at cryogenic temperatures into a range of optoelectronic devices of application in optical fibre communication, military systems, printing, infrared spectroscopy, pollution detection and short-pulse work, to mention only part of the list.

This excellent and comprehensive book opens with a most readable chapter outlining the history, concepts and basic principles, without the use of mathematics, emphasizing the dramatic effect of the introduction of hetero-structures, using layers of alloy material, which separately confine the injected carriers and guide the generated optical waves with favourable effects on threshold current and temperature of operation. In the next 100 pages the author discusses the basic processes of light emission and laser action, and includes appropriate development of absorption, stimulated and spontaneous processes, band structure in the presence of heavy doping, and recombination times. Gain-current relationships, threshold currents and efficiencies are calculated and compared with experimental results for the basic or homostructure $p-n$ junction laser with a simple resonator, for which the mode properties are also given. The treatment owes something to the earlier Semiconductor Lasers and Light Emitting Diodes by Kressel and Butler (Academic, 1977), but is more continuous and complete at the expense of rather intricate notation. I found this section a useful text for part of a graduate course on opte electronic devices held at the Optical Sciences Center of the University of Arizona.

Later chapters deal with heterostructure lasers, optical waveguides and all the many variations of laser geometries in considerable detail. Methods of fabrication and the physics of dynamical response are also considered.
The only notable omission is the absence of discussion in any detail of the lead salt family of diode lasers and their application in high resolution infrared spectroscopy, perhaps not surprising since the book concentrates on the GaAs alloys. However, the basic physical principles for all types of semiconductor diode lasers are well covered which is a stated aim of the book. The author does not claim to be comprehensive in citing the literature - a difficult task in this active field - but nevertheless provides copious references in each chapter.

The book frequently examines the relation between semiconductor lasers and other types of lasers - emphasizing the high gain values, for example. It is thus easy to see what semiconductor injection lasers have not achieved - visible room temperature operation, high powers or wide-band tuning in one device.

With the slight qualification that the book's title is not quite consistent with its concentration on the GaAs injection diode laser family, it is to be generally recommended for a variety of uses in research, application and teaching.

S. D. Smith is Visiting Professor at the Optical Sciences Center, University of Arizona, Tucson, and Head of the Physics Department at Heriot-Watt University, Edinburgh.

\section{Organic infrared-structure correlations}

\section{P.C. Crofts}

\section{Infrared Characteristic Group}

Frequencies. By G. Socrates. Pp.153. (Wiley: 1980.) £24, \$72.

INFRARED spectroscopy is perhaps the most generally applicable and widely used physical method for investigating structures of organic compounds. Although qualitatively rationalized by classical physical theory, it is an essentially empirical activity in which absorption bands reveal individual chemical bonds or larger submolecular units whose vibrations interact with radiation of the observed frequencies. Users of the technique thus require comprehensive collections of assignments of recorded absorption frequencies to particular functional groups or other structures. Although described in the preface as "a simple introduction to characteristic group frequencies", this book is a substantial and valuable work, simple only in its arrangement and empiricism and not in any restriction of its subject matter.

An introductory chapter contains three successively more detailed charts of the range $4000-200 \mathrm{~cm}^{-1}$; the first shows regions without absorptions and infers absences of particular groups, the second relates observed bands to possible vibrations, and the third shows absorption regions for many specified compound types. This is followed by 19 chapters dealing with individual substituents of particular importance (e.g. $\mathrm{O}-\mathrm{H}, \mathrm{C}=\mathrm{O}$ ), with collections of related substituents (e.g. triple-bonded groups, halogen compounds), or with larger structural features (e.g. alkane residues, sixmembered heterocycles). A short section surveys $\mathrm{X}-\mathrm{H}$ stretching overtones in the $14000-4000 \mathrm{~cm}^{-1}$ region. Each chapter contains extensive tables of absorption bands, in wavenumber and wavelength units, with well-written explanatory text and many references.

A compilation of this type can be only a starting point in an infrared investigation - which also requires comparison with reference spectra of model compounds but when used intelligently this book should point many investigators towards solution of their problems. It is well printed, with wide, short, double-column pages which are convenient when examining a spectrum, and is recommended to all who use infrared spectroscopy in structural investigations.

$\overrightarrow{P . C \text {. Crofts is a Senior Lecturer in Chemistry at }}$ the University of Manchester Institute of Science and Technology. 